\title{
Action des basses températures hivernales sur la photosynthèse du cèdre et du douglas
}

\author{
M. DUCREY (*) \\ avec la collaboration technique de P. Gross \\ I.N.R.A., Station de Sylviculture et de Production \\ Centre de Recherches forestieres de Nancy, \\ Champenoux, F 54280 Seichamps
}

\section{Résumé}

Pour étudier la photosynthèse hivernale du Cèdre (cedrus atlantica) et du Douglas (Pseudotsuga menziesii), on a utilisé des arbres de $2,5 \mathrm{~m}$ de hauteur cultivés en serre froide dans des conteneurs. La photosynthèse nette a été mesurée sur des rameaux rattachés à l'arbre et enfermés dans une enceinte d'assimilation régulée en température et en humidité. On a étudié la photosynthèse de ces rameaux pour des températures comprises entre $+10^{\circ} \mathrm{C}$ et $-8{ }^{\circ} \mathrm{C}$ tandis que la température de la serre variait de $+5^{\circ} \mathrm{C} \grave{a}+20^{\circ} \mathrm{C}$ selon les expériences.

Chez le douglas, la diminution de température du rameau de +10 " $\mathrm{C}$ à -6 " $\mathrm{C}$ n'affecte pas sensiblement le palier de photosynthèse. Par contre la photosynthèse augmente quand la température de la serre est plus élevée.

Chez le cèdre, on note une diminution très nette de la photosynthèse au-dessous de $-2^{\circ} \mathrm{C}$. Mais elle n'est pas encore nulle à $-8^{\circ} \mathrm{C}$. La respiration à l'obscurité est affectée dans les mêmes conditions. Au-dessus de 0 " $\mathrm{C}$, la réponse du cèdre dépend de la température de la serre, elle-même liée à l'optimum thermique des plants au cours de l'expérience.

Ces résultats sont comparés à ceux de la littérature ce qui a permis de mettre en évidence les modes d'action des basses températures. Elles ont tout d'abord une action directe à court terme sur le palier de photosynthèse, le rendement lunineux et la respiration à l'obscurité, qui aboutit à l'arrêt de la photosynthèse pour des températures voisines du point de congélation des tissus. Mais des arrière-effets sous forme d'inhibition temporaire de la photosynthèse sont constatés. Ces arrière-effets sorit cumulatifs et participent à l'installation d'une certaine adaptation au froid pendant la phase hivernale. Ces plants endurcis par le froid sont capables d'avoir en conditions naturelles une photosynthèse positive et, dans certains cas, un bilan photosynthétique positif pendant des périodes hivernales plus ou moins longues.

\section{1. - Introduction}

La persistance des aiguilles chez la plupart des conifères pendant l'hiver a posé, et pose encore le problème de leur fonctionnement photosynthétique en conditions hivernales et celui des gains de production liés à cette particularité.

(*) Adresse actuelle : Station de Bioclimatologie, I.N.R.A. Antilles-Guyane, 97170 Petit-Bourg, Guadeloupe (F.W.I.). 
Il est bien sûr difficile d'expliquer de cette manière une éventuelle supériorité de productivité des résineux par rapport aux feuillus, mais de nombreux chercheurs ont essayé de relier les gradients géographiques de production des espèces résineuses à des différences dans les conditions climatiques hivernales. C'est le cas par exemple de Pisex \& Winkler (1958) et de Tranquillini $(1957,1964)$ qui ont montré que la baisse de productivité de l'épicéa en altitude était liée à une augmentation de l'inactivité photosynthétique hivernale, elle-même dépendante du gradient thermique altitudinal.

La plupart des études concernent les aspects écophysiologiques et physiologiques de l'action des basses températures sur l'assimilation photosynthétique. Les expérimentations entreprises ont pour but d'analyser les différents processus physiologiques qui rentrent en jeu et de séparer les différents effets des basses températures. C'est ainsi que NeIrson et al. (1972) et Turner \& JARvis (1975) séparent l'effet de la température de l'air et de la température du sol, et mettent en évidence un effet d'adaptation progressive au froid. Une analyse assez complète de la résistance au froid pour les différents organes et les différentes fonctions des plantes est donnée par LARCHER et al. (1973).

Nous avons eu l'occasion d'aborder ce problème pendant les hivers 1976-1977 et 1977-1978 au cours desquels nous avons étudié la photosynthèse de jeunes cèdres et douglas placés en serre froide maintenue hors gel.

\section{2. - Matériel et méthode}

\subsection{Le matériel végétal}

Les arbres utilisés pour cette étude sont deux jeunes Cèdres (Cedrus atlantica Manetti) et un jeune Douglas (Pseudotsuga menziesii [Mirb.] Franco), âgés d'une dizaine d'années et hauts de $2,5 \mathrm{~m}$ environ. Ils sont élevés depuis 1975 dans des containers contenant $50 \mathrm{~kg}$ de terre et sont maintenus à un niveau de transpiration voisin de l'évapotranspiration potentielle pendant la saison de végétation. Ces arbres sont placés en permanence dans une serre qui limite la température à $25^{\circ} \mathrm{C}$ pendant la saison de végétation et qui reste hors-gel pendant l'hiver. La température y est alors comprise entre 3 et $15^{\circ} \mathrm{C}$ avec des pointes allant exceptionnellement jusqu'à $20^{\circ} \mathrm{C}$ suivant les conditions atmosphériques extérieures.

\subsection{La mesure de la photosynthèse nette}

La photosynthèse nette est mesurée dans une enceinte d'assimilation installée dans la serre. Le dispositif a fait par ailleurs l'objet d'une description détaillée (DUCREY, 1979) et nous nous bornerons à le décrire très succinctement.

L'enceinte d'assimilation fabriquée par Siemens est construite en plexiglas et a un volume utile de $38 \mathrm{~cm} \times 21 \mathrm{~cm} \times 7 \mathrm{~cm}$. La régulation de la température est effectuée au moyen de blocs Peltier et de sondes à résistance de platine. Le brassage 
interne de l'air assure une bonne régulation thermique et la puissance des blocs Peltier permet d'atteindre des températures de l'ordre de $\pm 20^{\circ} \mathrm{C}$ par rapport à la température ambiante. L'humidité est maintenue constante à l'entrée de l'enceinte et est régulée grâce à un système de piège à eau à point de rosée variable et de sondes à chlorure de lithium installé sur un circuit en dérivation. L'éclairage est assuré par la lumière naturelle de la serre et, pour les forts éclairements, par des lampes à vapeur de mercure de $2000 \mathrm{~W}$ (type MAZDA, MAF 2000). La photosynthèse nette est calculée grâce à un analyseur différentiel à infrarouge qui mesure la différence de concentration en $\mathrm{CO}_{2}$ de l'air entre l'entrée et la sortie de la chambre d'assimilation.

Au cours de nos expérimentations, un rameau était enfermé dans l'enceinte d'assimilation et soumis à un régime thermique déterminé tandis que le reste de l'arbre était à la température ambiante de la serre.

Les relations entre la photosynthèse nette et l'éclairement ont été établies pour différentes températures comprises entre $+10^{\circ} \mathrm{C}$ et $-8{ }^{\circ} \mathrm{C}$ alors que la température de la serre variait entre $+4^{\circ} \mathrm{C}$ et $+20^{\circ} \mathrm{C}$. Le rameau installé dans l'enceinte était soumis à la température choisie pendant toute la durée d'une manipulation, soit pendant près de 6 heures, et pendant le reste du temps il était remis à la température ambiante de la serre. Dans la suite de cet article, nous parlerons souvent, pour simplifier, de la température du rameau pour désigner la température de l'air de l'enceinte d'assimilation dans laquelle est placé le rameau.

Une première série de mesures a été faite du 9 février au 10 mars 1977 sur un cèdre et une seconde, du 26 janvier au 2 mars 1978, a permis d'étudier un douglas et un deuxième cèdre.

\section{3. - Résultats}

Les figures 1 et 2 montrent les courbes photosynthèse nette-éclairement qui ont été établies à différentes températures pour le douglas et le cèdre en février 1978. Les mesures de photosynthèse nette sont faites en lumière naturelle jusqu'à environ $50 \mathrm{~W} \mathrm{~m} \mathrm{~m}^{-2}$ tandis que l'éclairage additionnel a été utilisé pour les intensités lumineuses supérieures. Les courbes sont tracées par ajustement manuel à partir d'une cinquantaine de points expérimentaux. La dispersion des points expérimentaux qui n'ont pas pu être représentés sur les figures n'est pas très grande et est due en grande partie aux fluctuations de l'éclairage naturel de la serre. Ces courbes permettent de mettre en évidence la photosynthèse nette au palier de saturation observé entre 200 et $300 \mathrm{~W} \mathrm{~m}^{-2}$, le rendement lumineux, ainsi que la respiration à l'obscurité.

De manière générale, la température de l’enceinte est maintenue constante pendant toute la durée d'une expérience jusqu'à $0^{\circ} \mathrm{C}$ et même $-2{ }^{\circ} \mathrm{C}$. Au-dessous de ces valeurs, la température a tendance à augmenter au cours de l'expérience en raison de l'éclairage additionnel et de la température ambiante de la serre qui, vers midi, dépassait parfois $15^{\circ} \mathrm{C}$. Dans ces conditions, la différence de température entre l'enceinte et la serre atteint et dépasse $20^{\circ} \mathrm{C}$, et les éléments Pelticr ne sont pas suffisamment puissants pour assurer un refroidissement correct. 


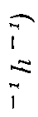

$i \frac{\vdots}{3}$

$0^{\circ} 0 \frac{0}{0}=\stackrel{0}{0}$

$=\sqrt{2}=\frac{1}{0}$ $\stackrel{\infty}{5} \frac{5}{2}$ i

$\bar{\Xi} \equiv$

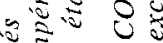

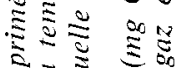

过 $\equiv$

$0_{2}=50$

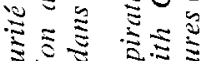

-

$2=0$ 离

出心

ए

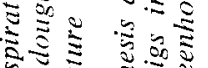

25:

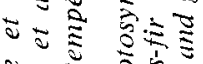

$\frac{5}{5} \pm \frac{5}{2}$

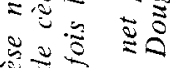

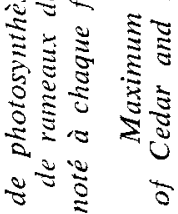

$\stackrel{5}{\Xi}=$

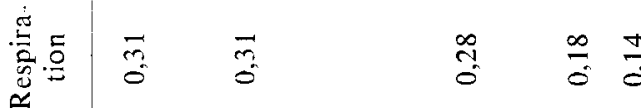

$\stackrel{0}{0}$

交

i $\frac{4}{2}$

ब्ञ

递

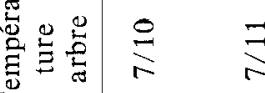

$\infty \quad \infty$ in

F

$\frac{0}{0}$

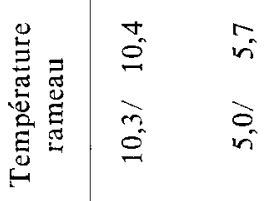

$\therefore \quad \begin{array}{lll}0 & \substack{n \\ j} & 1 \\ 0 & j & j \\ i & 1\end{array}$

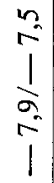

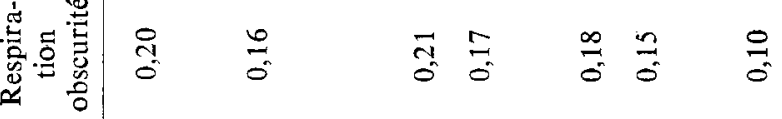

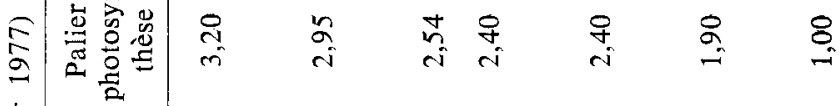

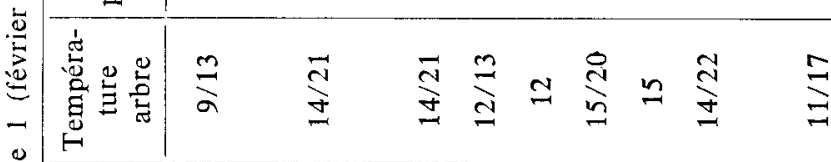

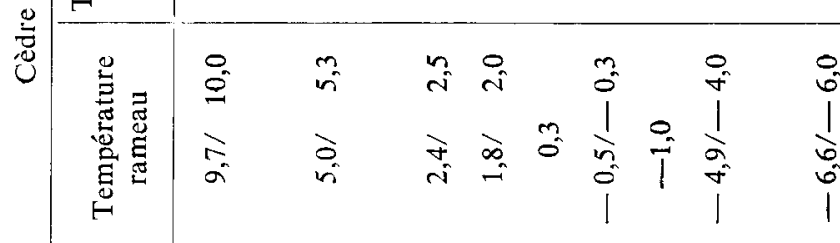

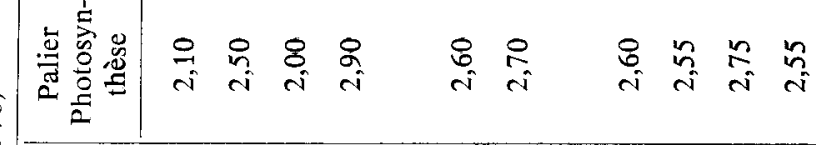

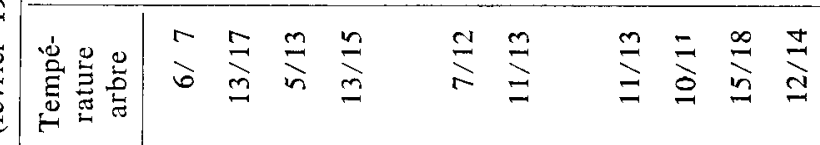

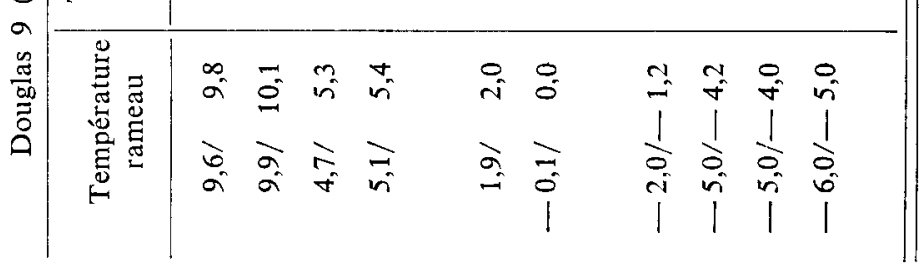




\section{Action de la température sur les paliers de saturation}

Pour le douglas, la variation de la température du rameau entre $+10^{\circ} \mathrm{C}$ et $-6^{\circ} \mathrm{C}$ ne paraît pas affecter significativement le palier de photosynthèse. Par contre, la température ambiante de la serre, donc celle de l'arbre dans son ensemble, a un effet plus direct. Ainsi, pour une même température de $10^{\circ} \mathrm{C}$, la photosynthèse nette atteint $2,1 \mathrm{mg} \mathrm{CO}_{2} \mathrm{~g}^{-1} \mathrm{~h}^{-1}$ quand la température ambiante est de 6 à $7^{\circ} \mathrm{C}$ et elle atteint $2,5 \mathrm{mg} \mathrm{CO} \mathrm{g}^{-1} \mathrm{~h}^{-1}$ quand la température ambiante se situe entre 13 et $17^{\circ} \mathrm{C}$. Des résultats analogues sont observés pour des températures de rameau de $+5{ }^{\circ} \mathrm{C}$ et $-5^{\circ} \mathrm{C}$ (voir aussi le tableau 1 ).

La photosynthèse des rameaux de cèdre paraît beaucoup plus affectée par la température de l'enceinte d'assimilation car elle passe de 3,2 à $1,0 \mathrm{mg} \mathrm{CO}_{2} \mathrm{~g}^{-1} \mathrm{~h}^{-1}$ pour le cèdre 1 et de 2,3 à $0,3 \mathrm{mg} \mathrm{CO} \mathrm{g}^{-1} \mathrm{~h}^{-1}$ pour le cèdre 3 quand la température diminue de $+10^{\circ} \mathrm{C}$ à $-8^{\circ} \mathrm{C}$.

Comme la température de la serre, pendant les expériences, varie entre 10 et $20^{\circ} \mathrm{C}$ pour le cèdre 1 et entre 5 et $10^{\circ} \mathrm{C}$ pour le cèdre 3 , on peut mettre en évidence l'effet de l'abaissement de la température du rameau sur sa photosynthèse, alors que le reste de l'arbre est dans une plage de température déterminée. C'est ce que montre la figure 3 . Pour le cèdre 1 , le palier de photosynthèse diminue régulièrement jusqu'à $-2{ }^{\circ} \mathrm{C}$ puis beaucoup plus rapidement au-dessous de $-2{ }^{\circ} \mathrm{C}$. La photosynthèse du cèdre 3 reste à peu près constante jusqu'à -2 "C et ensuite elle diminue très rapidement pour s'annuler vraisemblablement vers -8 ou $-9{ }^{\circ} \mathrm{C}$.

\section{Evolution des rendements lumineux}

La pente à l'origine des courbes photosynthèse nette-éclairement permet, sur les figures 1 et 2 de mettre en évidence le rendement lumineux.

Pour le douglas 9, on constate que la température du rameau au cours de l'expérience n'a pas d'influence sur le rendement lumineux. De même, les courbes de photosynthèse réalisées pour des températures ambiantes différentes suggèrent que jusque vers $15^{\circ} \mathrm{C}$ le rendement lumineux n'est pas affecté, mais qu'il augmente quand la température de l'ensemble de l'arbre se situe entre 15 et $18^{\circ} \mathrm{C}$. Pour le cèdre 3 , le rendement lumineux évolue dans le même sens que les paliers de saturation correspondants.

Toutefois, il n'est pas possible de pousser plus en avant l'interprétation des données de rendement lumineux car celles-ci sont simplement déduites des courbes photosynthèse nette-éclairement, et n'ont pas été mesurées directement.

\section{Evolution de la respiration à l'obscurité}

La respiration à lobscurité a pu être mesurée uniquement pour le cèdre. On constate, sur le tableau 1, que la respiration à l'obscurité est affectée par la température du rameau. Elle est en effet de $0,3 \mathrm{mg} \mathrm{CO}_{2} \mathrm{~g}^{-1} \mathrm{~h}^{-1}$ pour le cèdre 3 et de $0,2 \mathrm{mg} \mathrm{CO}_{2} \mathrm{~g}^{-1} \mathrm{~h}^{-1}$ pour le cèdre 1 quand la température du rameau est de $10^{\circ} \mathrm{C}$, et elle diminue progressivement pour atteindre $0,1 \mathrm{mg} \mathrm{CO}_{2} \mathrm{~g}^{-1} \mathrm{~h}^{-1}$ vers -6 et $-8^{\circ} \mathrm{C}$. 


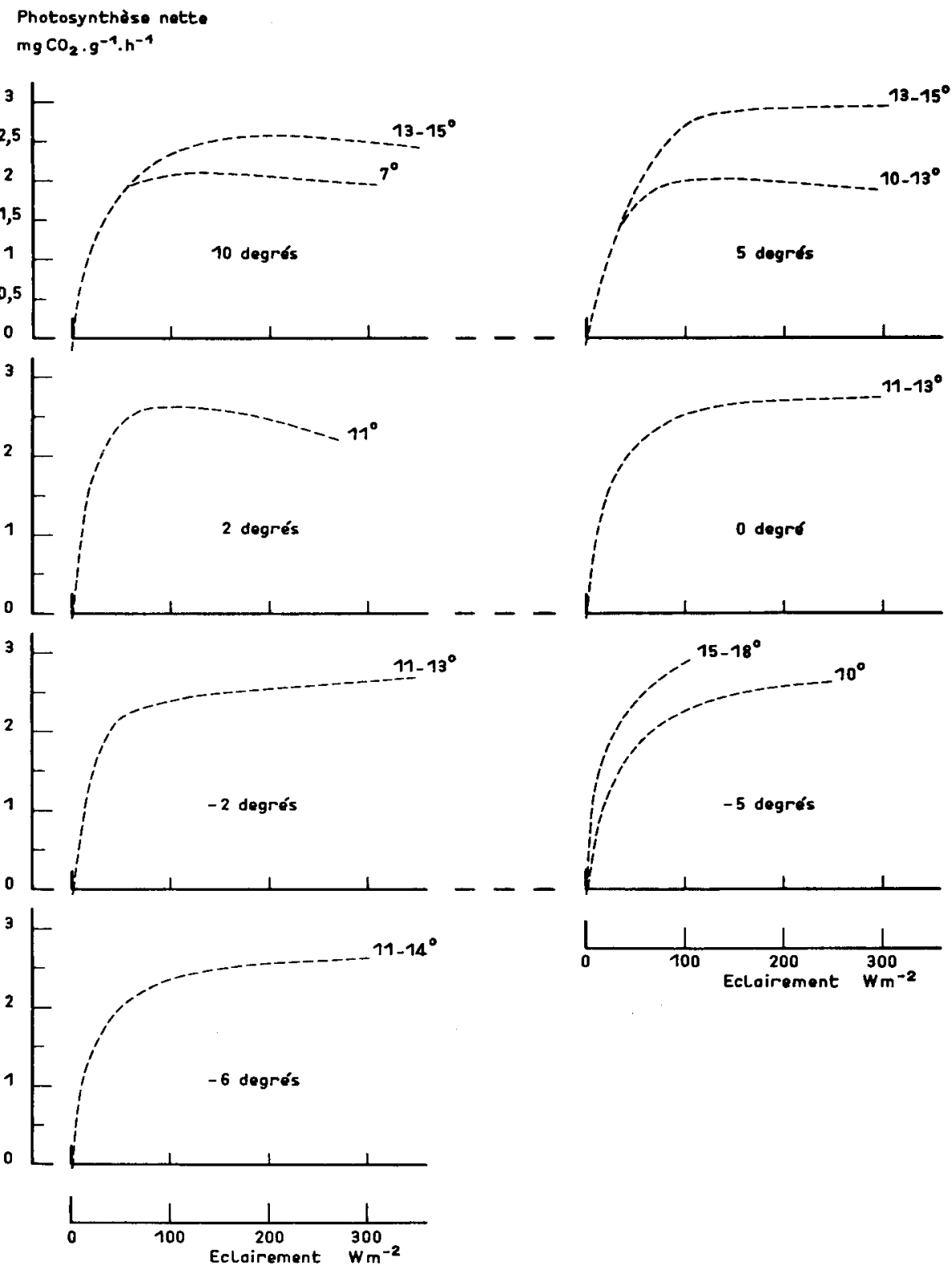

FIG. 1

Courbes photosynthèse nette-éclairement d'un rameau de douglas rattaché à l'arbre, pour différentes températures échelonnées entre $+10^{\circ} \mathrm{C}$ et $-6^{\circ} \mathrm{C}$.

On a noté en face de chaque courbe la température de la serre au moment de l'expérience (douglas 9, février 1978)

The relation between photosynthesis and light intensity for Douglas-fir twig on the tree, at different temperatures varying from $+10^{\circ} \mathrm{C}$ to $-6^{\circ} \mathrm{C}$

Greenhouse temperature during the experiment is registered on each curve (Douglas-fir 9, february 1978) 

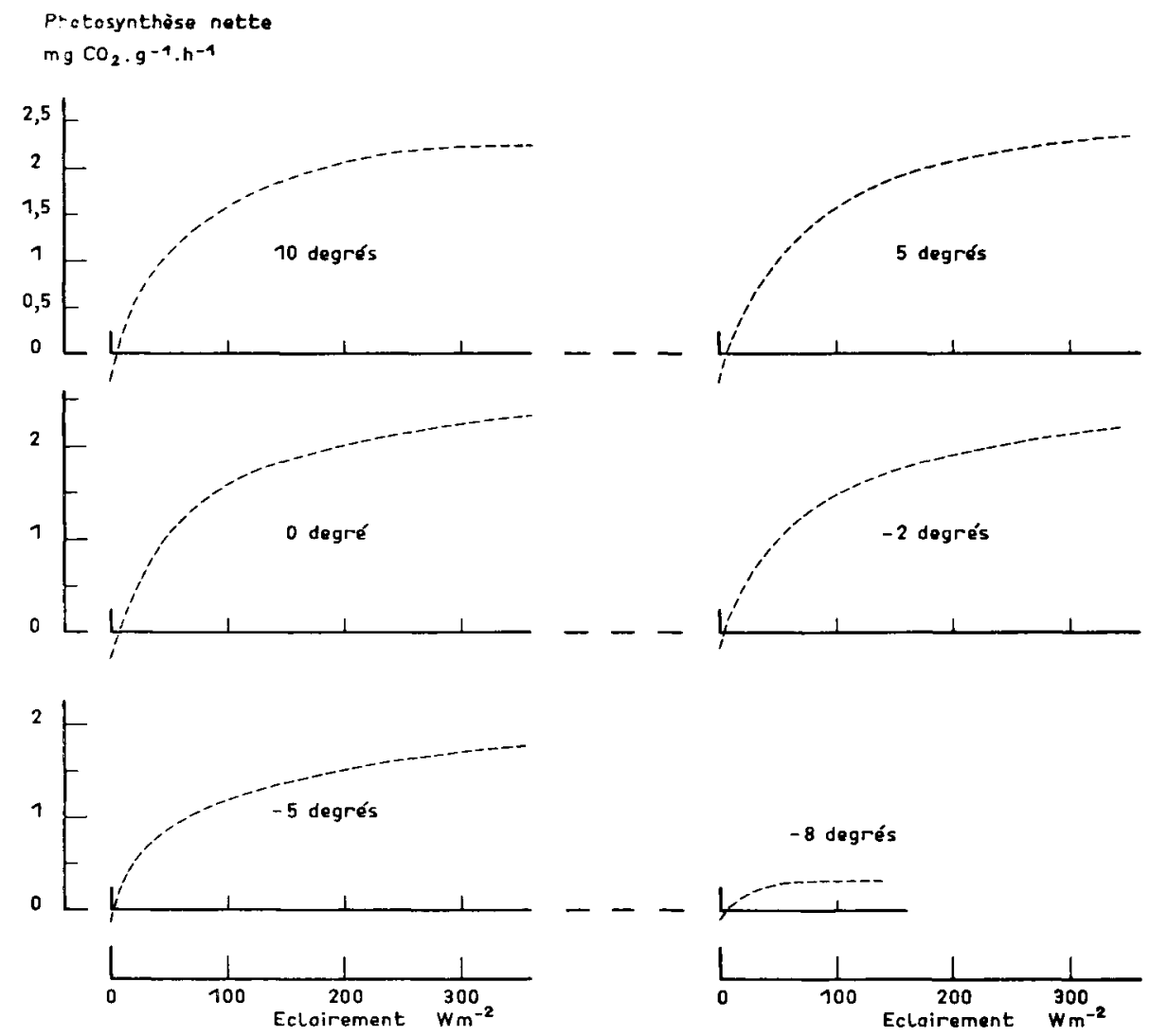

Fig. 2

Courbes photosynthèse nette-éclairement d'un rameau de cèdre rattaché à l'arbre, pour différentes températures échelonnées entre $+10^{\circ} \mathrm{C}$ et $-8^{\circ} \mathrm{C}$

La température de la serre au moment de l'expérience variait entre $4^{\circ} \mathrm{C}$ et $11^{\circ} \mathrm{C}$ (cèdre 3, février 1978)

The relation between net photosynthesis and light intensity for Cedar twig on the tree, at different temperatures varying from $+10^{\circ} \mathrm{C}$ to $-8{ }^{\circ} \mathrm{C}$.

Greenhouse temperature was varying between $+4^{\circ} \mathrm{C}$ and $+11^{\circ} \mathrm{C}$ during the experiment (Cedar 3, february i978)

\section{4. - Discussion et conclusion}

La difficulté est toujours grande d'effectuer des mesures de photosynthèse à des températures négatives et nous avons rencontré de nombreux problèmes d'ordre méthodologique et instrumental. En particulier se posent des problèmes de condensation sous forme d'eau et même de glace notamment dans les pièges à eau et aussi des problèmes d'étalonnage liés à la faible mais variable teneur en eau de l'air traversant l'analyseur de gaz carbonique. C'est ce qui nous a amenés à réguler la 
température uniquement pendant les 5 à 6 heures que dure une expérience et à laisser pendant le reste du temps, l'ensemble de l'appareillage à la température ambiante de la serre.

Dans ces conditions, l'arbre entier est toujours à la température ambiante de la serre : on peut alors estimer qu'il est physiologiquement adapté à photosynthétiser de manière optimale dans ces conditions de température. Si alors on fait photosynthétiser cet arbre à des températures variables, on peut analyser ses réactions à ces basses températures passagères. On peut aussi étudier l'effet de l'adaptation à une température donnée sur la photosynthèse aux basses températures.

Dans notre expérience, cette interprétation doit être plus nuancée car le rameau soumis aux basses températures reste attaché à l'arbre qui lui, est à la température de la serre. Ceci peut avoir pour effet d'accélérer les transferts d'assimilats entre le rameau et le reste de l'arbre, donc d'augmenter les capacités de photosynthèse du rameau. Il est cependant difficile de donner l'importance exacte de ce phénomène qui est lui-même fortement limité par le fait que les aiguilles sont séparées du reste de l'arbre par la portion du rameau - une vingtaine de centimètres — située dans l'enceinte d'assimilation et soumise à la même température que les aiguilles.

\subsection{Action directe de la température sur la photosynthèse nette}

Nous avons vu pour le cèdre que la photosynthèse est peu ou pas influencéc par la température jusqu'à $\longrightarrow 2$ "C. Ensuite, elle diminue très rapidement pour s'annuler vraisemblablement vers -8 ou $-9^{\circ} \mathrm{C}$. BAUER et al. (1975) indiquent que l'entrée du $\mathrm{CO}_{2}$ diminue brusquement lorsque les organes photosynthétiques commencent à geler. Les raisons de cette interruption de l'entrée du $\mathrm{CO}_{2}$ sont la fermeture des stomates induite par le gel, laccroissement rapide de la résistance dans le mésophylle en raison de la formation de glace dans les espaces intercellulaires et enfin, l'effet du gel extracellulaire sur l'activité enzymatique et l'ultrastructure des chloroplastes. PISEK et al. (1967) donnent comme limites inférieures pour la photosynthèse hivernale : $-7{ }^{\circ} \mathrm{C}$ pour Pinus sylvestris, $-7,5^{\circ} \mathrm{C}$ pour Albies alba et $-6,5^{\circ} \mathrm{C}$ pour Picea abies. La limite de $-8{ }^{\circ} \mathrm{C}$ que nous trouvons pour le Cèdre est du même ordre de grandeur.

Pour le douglas, nous n'avons pas noté de diminution de la photosynthèse jusqu'à $-6{ }^{\circ} \mathrm{C}$, ce qui permet de penser que la température de gel des tissus n'est pas encore atteinte. Elle doit vraisemblablement être inférieure à celle du cèdre.

Il faut préciser que ces températures correspondent à des arbres adaptés aux conditions hivernales. Pour des arbres en phase de végétation, le gel des organes photosynthétiques donc l'arrêt de la photosynthèse se produit à des températures plus élevées, de l'ordre de -3 à $-5^{\circ} \mathrm{C}$ (PISEK et al., 1967).

En ce qui concerne la respiration à l'obscurité, nos résultats montrent qu'elle diminue très lentement mais qu'elle n'est pas encore nulle à $-8{ }^{\circ} \mathrm{C}$. Ceci est conforme aux résultats de BaUER et al. (1969) sur Abies alba, qui trouvent que la respiration persiste quand les tissus sont gelés et que, au moment du dégel, elle est très fortement stimulée. 


\subsection{Les arrière-effets des basses températures}

On peut se poser la question concernant nos expériences, de savoir si les températures négatives appliquées au rameau pendant plusieurs heures sont susceptibles de modifier son comportement les jours suivants. BAuER et al. (1969) montrent qu'audessus du point de gelée des aiguilles de Abies alba qui est de -4 "C en octobre et de -8 " $\mathrm{C}$ en décembre, la photosynthèse mesurée à $15^{\circ} \mathrm{C}$ au bout de 24 heures n'est pas modifiée par le passage à basse température. Des résultats analogues de Pisek \& Kemnitzer (1968) indiquent que des plants de Abies alha adaptés au froid peuvent être placés à $-5^{\circ} \mathrm{C}$ pendant 48 heures et retrouver au bout de 24 heures une photosynthèse normale.

$\mathrm{Si}$ on se rappelle que les tissus de Abies alba gèlent vers $-7,5^{\circ} \mathrm{C}$, on peut estimer que le cèdre et le douglas ont un comportement à peu près analogue à Albies alba et que, au cours de nos expériences, les capacités photosynthétiques des rameaux nont pas été altérées par le passage intermittent à des températures négatives.

L'étude de ces arrière-effets des basses températures est très importante car elle permet de tester l'endurcissement des arbres au froid et leur adaptation à photosynthétiser après des passages plus ou moins prolongés au froid.

\subsection{Photosynthèse et adaptation aux basses températures}

L'adaptation aux basses températures peut être caractériséc par les possibilités que possèdent les arbres à feuilles persistantes de photosynthétiser en hiver à un taux non négligeable par rapport à la photosynthèse estivale, et par la faculté qu'ont ces arbres à récupérer très rapidement une photosynthèse normale après passage à des températures voisines du point de congélation de leurs tissus.

Des études, faites pendant l'été 1977 sur des arbres provenant des mêmes lots expérimentaux que ceux utilisés pour nos expériences hivernales, ont permis de tracer les courbes photosynthèse nette-éclairement et de calculer les paliers de saturation correspondants. On constate que, pendant la période estivale la photosynthèse est environ de $4 \mathrm{mg} \mathrm{CO}_{2} \mathrm{~g}^{-1} \mathrm{~h}^{-1}$ pour le douglas et de $4,5 \mathrm{mg} \mathrm{CO} . \mathrm{g}^{-1} \mathrm{~h}^{-1}$ pour le cèdre. Ces chiffres sont à rapprocher de ceux obtenus pendant la période hivernale et on peut dire globalement que la photosynthèse en hiver, mesurée à la température ambiante de la serre est environ les deux tiers de la photosynthèse d'été. L'abaissement de température n'est d'ailleurs pas le seul responsable de cette diminution de la photosynthèse car il faut tenir compte aussi du vieillissement des aiguilles (EL Aouni \& Mousseau, 1974 ; Kunstle \& Mitscherlich, 1975). Des résultats plus précis concernant le cèdre 1 montrent que sa photosynthèse était de $5 \mathrm{mg} \mathrm{CO}: \mathrm{g}^{-1} \mathrm{~h}^{\cdots 1}$ au début juillet 1977, de $4,4 \mathrm{mg} \mathrm{CO}_{2} \mathrm{~g}^{-1} \mathrm{~h}^{-1}$ au début août et de $3,8 \mathrm{mg} \mathrm{CO}_{2} \mathrm{~g}^{-1} \mathbf{h}^{-1}$ au début septembre. Comme nous l'avons vu, elle est encore de $3 \mathrm{mg} \mathrm{CO}_{2} \mathrm{~g}^{-1} \mathrm{~h}^{-1}$ au début février 1978. Ceci correspond à une température de la serre voisine en moyenne de $15^{\circ} \mathrm{C}$.

La figure 3 montre que la photosynthèse du cèdre 1 diminue entre $10^{\circ} \mathrm{C}$ et $0^{\circ} \mathrm{C}$ ce qui s'explique si on admet que le cèdre 1 est temporairement adapté à fonctionner à $15^{\circ} \mathrm{C}$ donc qu'il est alors à son optimum thermique. Par contre, les expérimentations ont été faites sur le cèdre 3 lorsque la température de la serre 
était plus basse, $7{ }^{\circ} \mathrm{C}$ environ, ce qui explique le palier de photosynthèse observé entre $0{ }^{\circ} \mathrm{C}$ et $10^{\circ} \mathrm{C}$. On remarquera aussi que les deux cèdres ont un comportement identique au-dessous de $0^{\circ} \mathrm{C}$ et il semble que dans ce cas l'action directe de la température l'emporte sur l'effet adaptatif. NeILson et al. (1972) arrivent avec Picea sitchensis à des conclusions analogues.

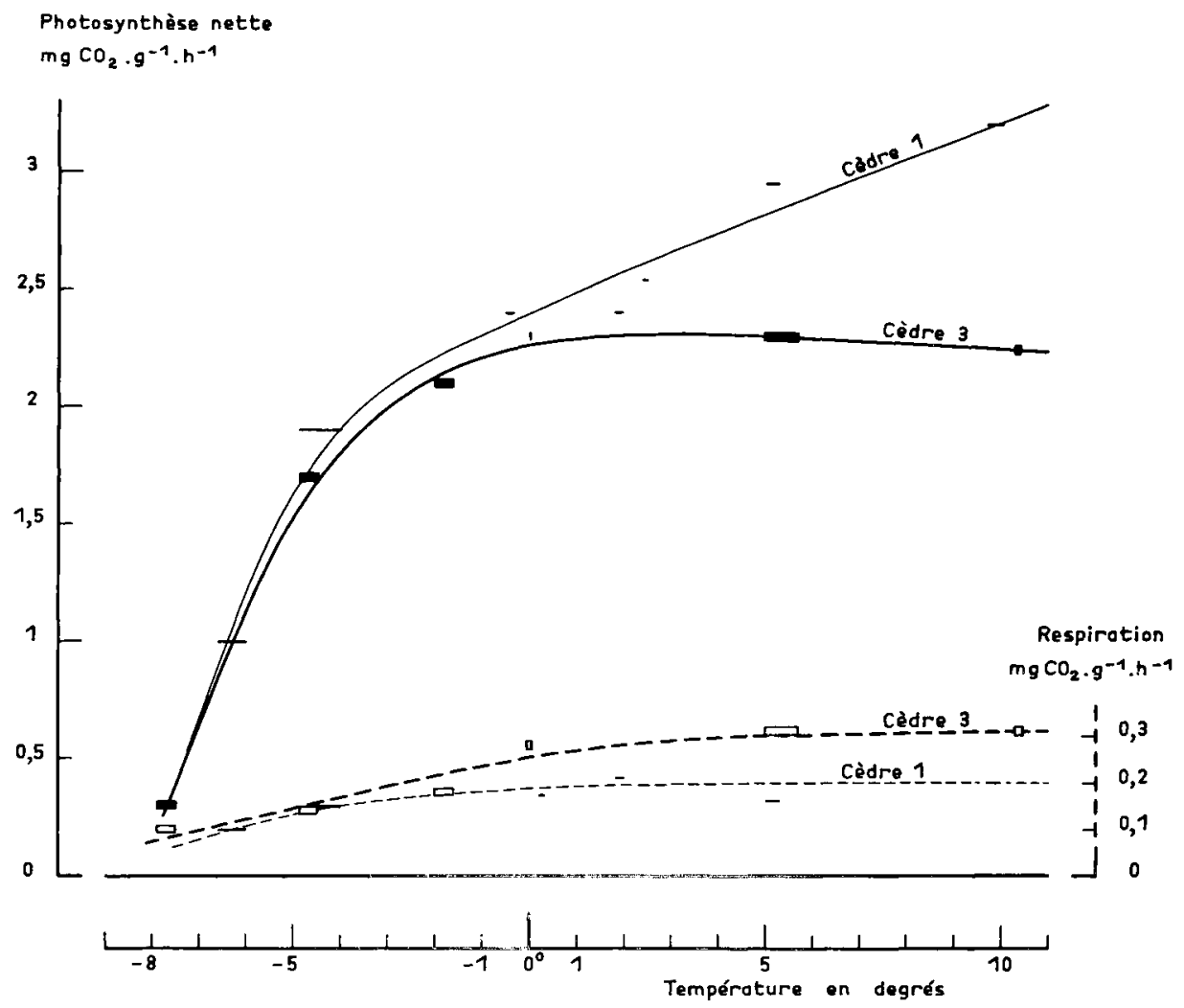

FIG. 3

Action de la température sur le palier de photosynthìse nette et sur la respiration à l'obscurité pour 2 rameatix de ciedre.

Pendant les expériences sur le cèdre l la température de la serre oscillait entre $10^{\circ} \mathrm{C}$ et $20^{\circ} \mathrm{C}$. Pour le cedre 3 elle variait entre $4{ }^{\prime \prime} \mathrm{C}$ et $11^{\circ} \mathrm{C}$

Effects of temperature on maximum net photosynthesis and dark respiration for two Cedar twigs.

During the experiments greenhouse temperature was varying from $10^{\circ} \mathrm{C}$ to $20^{\circ} \mathrm{C}$ for Cedar 1 and from $4{ }^{\circ} \mathrm{C}$ to $11{ }^{\circ} \mathrm{C}$ for Cedar 3

L'adaptation aux basses températures se traduit aussi par la possibilité de résister sans dommages à des températures très nettement négatives.

Pour Picea sitchensis, NeILson et al. (1972) montrent que la photosynthèse de plants non adaptés au froid est très fortement inhibée par le passage à $-5^{\circ} \mathrm{C}$ pen- 
dant une heure tandis que les plants parfaitement adaptés au froid ne sont pas affectés par ce passage à basse température. Pour Abies alba, Pisek \& Kemnitzer (1968) ont observé que des plants relativement peu adaptés au froid - plants prélevés en forêt en octobre - nécessitaient un passage à $-8^{\circ} \mathrm{C}$ pendant 12 heures pour que la photosynthèse soit encore nulle dans les conditions normales 24 heures plus tard. Pour des plants très bien adaptés au froid — prélevés en forêt en février-mars - la récupération de la photosynthèse est complète 24 heures après un passage à $-14^{\circ} \mathrm{C}$ pendant 12 heures. Cette récupération est encore de 65 p. 100 si la température est descendue jusqu'à - 18 " $\mathrm{C}$.

Ces deux exemples montrent le caractère progressif de l'adaptation au froid, les arbres présentant pendant l'hiver une meilleure adaptation au froid et une meilleure résistance aux basses températures qu'au moment des premiers froids. Ils montrent aussi les différences de comportement entre 2 espèces, l'une océanique : Picea sitchensis et lautre plus continentale et adaptée aux conditions de montagne : Abies alba.

Bien que nous n'ayons pas poussé aussi loin les expériences d'endurcissement au froid du cèdre et du douglas, nous pouvons cependant constater la supériorité du douglas sur le cèdre. Ceci est en accord avec le caractère méditerranéen bien que montagnard du cèdre, tandis que le douglas est adapté à des climats tempérés possédant des hivers plus rigoureux.

\subsection{Photosynthèse hivernale en conditions naturelles}

Les études en conditions contrôlées donnent des résultats très tranchés concernant l'adaptation et les limites de la résistance au froid. Dans la nature, les résultats ne sont pas aussi nets mais au contraire beaucoup plus nuancés.

NeILsON et al. (1972) montrent le caractère cumulatif de l'acquisition de la résistance au froid. Ils trouvent qu'en forêt, les jeunes pousses de Picea sitchensis possèdent encore en juillet et août un certain endurcissement au froid, caractère qui disparaît en septembre et qui réapparaît très rapidement en 2 ou 4 semaines en novembre à la suite de nombreuses gelées très fortes. Cette acquisition de l'endurcissement au froid s'accompagne d'un abaissement de l'optimum thermique. Mais le caractère d'endurcissement au froid ne reste pas constant pendant tout l'hiver.

C’est ainsi que Gueht (1978) montre que le douglas en région parisienne garde en mémoire les antécédents climatiques pendant toute une semaine. L'effet sur la photosynthèse est par conséquent très atténué ou au contraire amplifié en fonction des séquences climatiques antérieures.

Cependant, dans les conditions naturelles, les températures négatives sont accompagnées d'un sol gelé. C'est pourquoi Schmidt-Vogt \& Gross (1976) montrent que le bilan photosynthétique journalier de l'épicéa est positif quand la température de lair est positive. Mais il peut être négatif dans les mêmes conditions quand le sol est encore gelé. C'est alors le stress hydrique créé par le gel qui entraîne l'interruption de la photosynthèse. Mais les températures négatives ont aussi un effet direct sur le système racinaire. C'est ce qu'ont montré TURNER \& JARvis (1975) en analysant la réponse photosynthétique de Picea sitchensis à des températures de sol descendant jusqu'à $-8{ }^{\circ} \mathrm{C}$. Ils observent une nette diminution de la photosynthèse à partir de 
$+1{ }^{\circ} \mathrm{C}$ pour des plants non endurcis et de $-1{ }^{\circ} \mathrm{C}$ pour des plants endurcis. Pour des températures de sol négatives, la vitesse de récupération des capacités photosynthétiques dépend à la fois de la température et du degré d'endurcissement des plants.

Dans ces conditions on conçoit que le bilan photosynthétique hivernal qui dépend de très nombreux facteurs internes et externes à l'arbre, va être différent suivant les espèces.

Pour Pinus aristata, Schulze et al. (1967) trouvent un bilan hivernal très nettement négatif parce que la photosynthèse s'annule complètement pendant l'hiver et ne réagit pas aux courtes périodes chaudes hivernales, tandis que la respiration reste à un taux très élevé. Tranquillini (1957) observe chez Pinus cembra un bilan négatif continu pendant 5 mois pour les individus situés à la limite supérieure altitudinale de l'espèce.

D'autres auteurs et parmi eux Freeland (1944), Zeller (1951) et Parker (1953, 1961) mesurent pour diverses espèces forestières des bilans négatifs pendant de plus ou moins longues périodes hivernales mais estiment que sur l'ensemble de l'hiver, les gains photosynthétiques compensent les pertes respiratoires.

Nous n'avons pas trouvé d'auteurs signalant des bilans franchement positifs. Il est cependant probable, à la lumière de ce qui a été dit au cours de cet article, que de tels bilans sont possibles, notamment en situation de plaine ou sous des climats relativement océaniques.

Reçu pour publication en aồt 1980.

\author{
Summary \\ Effects of low wintertime temperatures \\ on photosynthesis of Cedar and Douglas-fir
}

For studies of the wintertime photosynthesis of Cedar (Cedrus atlantica) and Douglas-fir (Pseudotsuga menziesii), we used some 2.5 meter high trees grown in a cold greenhouse. Net photosynthesis of twigs bound with the tree was measured in a $\mathrm{CO}_{\mathrm{z}}$-gaz exchange chamber, regulated in temperature and air humidity. Twigs photosynthesis measurements were made for temperature between $+10^{\circ} \mathrm{C}$ and $-8{ }^{\prime \prime} \mathrm{C}$ meanwhile the greenhouse air temperature was varying from $+5^{\circ} \mathrm{C}$ to $+20^{\circ} \mathrm{C}$ according to experiments.

For Douglas-fir, a decrease of the twig temperature from $+10^{\circ} \mathrm{C}$ to $-6^{\circ} \mathrm{C}$ has no apparent effect on maximum net photosynthesis. On the other hand, photosynthesis increases when the greenhouse air temperature is higher.

For Cedar, net photosynthesis is decreasing under -2 " $\mathrm{C}$, but is still not equal to zero for -8 " $\mathrm{C}$. Dark respiration is affected in the same conditions. Over $0{ }^{\circ} \mathrm{C}$, the reaction of Cedar is related with the greenhouse temperature.

These results are compared to these ones from the literature and point out the effects of low temperatures. Low temperatures have a short-term effect on maximum net photosynthesis, on maximum efficiency of light energy conversion, and on dark respiration. Then, photosynthesis decreases and stops for temperatures near of the tissue freezing point. But after-effects of low temperatures are observed as temporary inhibition of photosynthesis. These after-effects are a cumulative process and produce frost hardiness. These hardened plants may have, in natural conditions, a positive net photosynthesis and, in some cases. a positive photosynthetic balance during more or less large winter periods. 


\section{Références bibliographiques}

Bauer H., Huter M., Larcher W., 1969. Der Einfluss und die Nachwirkung von Hilze und Kältestress auf den $\mathrm{CO}$-Gaswechsel von Tanne und Ahorn. Ber. dtsch. bot. Ges., 82. 65-70.

Bauer H., Larcher W., Walker R.B., 1975. Influence of temperalure on CO-gaz exchange in : Photosynthesis and productivity in different environments, pp. 557-586, I.B.P., vol. 3, Cambridge Univ. Press.

Ducrey M., 1979. Description d'une enceinte régulée en température, humidité et gaz carbonique pour l'étude de la photosynthèse, de la respiration et de la transpiration. Publication Station de Sylviculture, C.N.R.F.-I.N.R.A., 25 p.

El Aouni M.H., Moussenu M., 1974. Relation d'échange de $\mathrm{CO}_{2}$ chez les aiguilles du Pin noir d'Autriche (Pinus nigra Arn.) avec l'âge, la teneur en chlorophylle et la réassimilation. Photosynthetica. 8 (2), 78-86.

Freeland R.O., 1944. Apparent photosynthesis in some conifers during winter. Plant Physiol., 19, $179-185$.

Gcehl J.M., 1978. Aspect hivernal du fonctionnement photosynthétique du Douglas. D.E.A. de Physiologie Végétale, Université Paris VII, $44 \mathrm{p}$.

Kunstle E. Mitscherlich G., 1975. Photosynthese, Transpiration und Atmung in einem Mischbestand im Schwarzwald. I. Teil : Photosynthese. Allg. Forst-ll J.-Zig., 146. 45-63.

Larcher W., Heber U., Santarius K.A., 1973. Limiting temperature for life functions. In : Temperature and life, pp. 195-231, Precht $\mathrm{H}$. ct al. Ed., Springer-Verlag, Berlin.

Neilson R.E., Ludlow M.M., Jarvis P.G., 1972. Photosynthesis in Sitka spruce (Picea sitchensis [Bong.] Carr.). II. Response to temperature. J. appl. Ecol., 9, 721-745.

Parker J., 1953. Photosynthesis of Picea excelsa in Winter. Ecology, 34, 605-609.

Parker J., 1961. Seasonal trends in carbon dioxide absorption, cold resistance and transpiration in some evergreens. Ecology, 42, 372-380.

Pisek A., Kemnitzer R., 1968. Der Einfluss von Frost auf die Photosynthese der Weisstanne (Abies alba Mill.). Flora, B 157, 314-326.

Pisek A.. Larcher W., Unterholzaner R., 1967. Kardinale Temperaturbereiche der photosynthese und Grenztemperaturen des lebens der Blätter verschiedenen Spermatophyten. I. Temperaturminimum der nettoassimilation, Gefrier - Und Frostchadenbereiche der Blätter. Flora, B 157, 239-264.

PISEK A., Winkler E., 1958. Assimilationsvermögen und Respiration der Fichte (Picea excelsa link.) in verschiedenen Höhenlagen und der Zirbe (Pinus cembra L.) an der alpinen Waldgrenze. Planta, 51, 518-543.

Schmidt-Vogt H., Gross K., 1976. Untersuchungen zum winterlichen Gaswechsel der Fichte (Picea abies [L.] Karst.) unter Freilandbedingungen. All. Forst-ll. J.-Ztg., 147, 189-192.

SChulze E.D., Mooney H.A., Dunn E.I., 1967. Wintertime photosynthesis of Bristlcone pine (Pinus aristata) in the White Mountains of California. Ecology, 48, 1044-1047.

Tranquillini W. 1957. Photosynthesis and dry matter production of trees at high altitude, p. 501-508. In : Formation of wood in forest trees, Academic Press, New York.

Tranquillini W., 1964. The physiology of plants at high altitude. Anmu. Rev. Plant Physiol., 15, 345-362.

Turner N.C., Jarvis P.G., 1975. Photosynthesis in Sitka Spruce (Picea sitchensis [Bong.] Carr.). IV. Response to Soil temperature. J. appl. Ecol., 12, 561-576.

Zeller O., 1951. Uber Assimilation und Atmung der Pflanzen im winter bei tiefen Temperaturen. Planta, 39, 500-526. 